This is the accepted version of the following article:

Delgado-Pérez, P, Sánchez, AB, Segura, S and Medina-Bulo, I. Performance Mutation Testing. Softw Test Verif Reliab. 2019;

e1728. https://doi.org/10.1002/stvr.1728

which has been published in final form at:

https://onlinelibrary.wiley.com/doi/abs/10.1002/stvr.1728

This article may be used for non-commercial purposes in accordance with the Wiley SelfArchiving Policy [ http://www. wileyauthors.com/self-archiving]. 


\title{
Performance Mutation Testing
}

\author{
Pedro Delgado-Pérez ${ }^{1 *}$, Ana B. Sánchez ${ }^{2}$, Sergio Segura ${ }^{2}$, Inmaculada Medina-Bulo $^{1}$ \\ ${ }^{1}$ Universidad de Cádiz, Escuela Superior de Ingeniería, Spain. \\ ${ }^{2}$ Universidad de Sevilla, ETS Ingeniería Informática, Spain.
}

\begin{abstract}
SUMMARY
Performance bugs are known to be a major threat to the success of software products. Performance tests aim to detect performance bugs by executing the program through test cases and checking whether it exhibits a noticeable performance degradation. The principles of mutation testing, a well-established testing technique for the assessment of test suites through the injection of artificial faults, could be exploited to evaluate and improve the detection power of performance tests. However, the application of mutation testing to assess performance tests, henceforth called performance mutation testing (PMT), is a novel research topic with numerous open challenges. In previous papers, we identified some key challenges related to PMT. In this work, we go a step further and explore the feasibility of applying PMT at the source-code level in generalpurpose languages. To do so, we revisit concepts associated with classical mutation testing, and design seven novel mutation operators to model known bug-inducing patterns. As a proof of concept, we applied traditional mutation operators as well as performance mutation operators to open-source $\mathrm{C}++$ programs. The results reveal the potential of the new performance-mutants to help assess and enhance performance tests when compared to traditional mutants. A review of live mutants in these programs suggests that they can induce the design of special test inputs. In addition to these promising results, our work brings a whole new set of challenges related to PMT, which will hopefully serve as a starting point for new contributions in the area. Copyright $(\odot) 0000$ John Wiley \& Sons, Ltd.
\end{abstract}

Received ...

KEY WORDS: Software testing, mutation testing, performance testing, performance bugs.

\section{INTRODUCTION}

Performance bugs are programming errors that can cause a significant performance degradation like excessive memory consumption [1] or energy leaks [2,3]. Performance bugs affect to key nonfunctional properties of programs such as execution time or memory consumption. These types of bugs are very harmful and common in released software programs [4], causing poor usability and waste of resources. This might lead to a loss of users, loss of money or, in the worst case, loss of human lives, e.g., a self-driving car not braking in time. For example, recent studies [5, 6] show that

\footnotetext{
${ }^{*}$ Correspondence to: Escuela Superior de Ingeniería, Avda. de la Universidad de Cádiz 10, 11519 Campus Universitario de Puerto Real, Spain. Email: pedro.delgado@uca.es 
$40 \%$ of the users will leave a website that takes more than 3 seconds to load, $8 \%$ of them will stop a purchase in a poor performing site, or $79 \%$ will be less likely to purchase again on the same site if they are dissatisfied with their visit in terms of performance. Therefore, performance bugs require full attention to increase the loyalty of users and the safety of systems.

Compared to functional faults, performance bugs are significantly harder to detect and require more time and effort to be fixed [3]. This is partly due to the lack of test oracles, that is, mechanisms to decide whether the performance of the program with a given input is acceptable i.e., the oracle problem [7, 8]. For instance, Nistor et al. [3] analyzed 210 performance bugs from three mature open source projects and concluded that "better oracles are needed for discovering performance bugs". In contrast to functional bugs, performance bugs do not usually produce wrong results or crashes in the program under test and therefore they cannot be detected by simply inspecting the program output. Furthermore, performance indicators are by nature non-deterministic and can vary among executions due to numerous factors, such as the device hardware, configuration settings, or the current workload [9]. For example, suppose a mobile shopping app that consumes 30Mb of memory: Is this the expected performance? How much memory should it be considered as a performance bug? These types of questions are typically answered by using execution profilers [1, 10,11] or performance tests $[12,13]$. Performance bugs can also be detected by searching for bug-inducing patterns identified in previous versions of the target or related programs [7, 14, 15, 16]. Applying performance tests is crucial to gain confidence that the developed systems are free of performance faults, that is, deviations with respect to the specification in terms of performance. Note that this is different from program optimization [2,17], whose goal is to improve the performance of a program to achieve a less consuming version. Despite the relevance of this issue, we have identified a lack of mechanisms to guide the assessment and improvement of performance tests.

Mutation testing is a fully-fledged testing technique for the assessment and improvement of test suites. In mutation testing, multiple faulty versions of the target program, so-called mutants, are generated by applying mutation operators. Each mutant is generated by applying a syntactic change to the original program. Those mutants are then executed against the test suite to evaluate its faultrevealing capability. Some mutants may not change the semantics of the program and are called semantically-equivalent mutants. Telling whether a mutant is equivalent is an undecidable problem, which currently represents one of the major obstacles for the wide-spread adoption of mutation testing [18]. Catalogs of mutation operators have been proposed in most popular programming languages. However, mutation testing has mostly been applied for functional testing, remaining its applicability to performance testing as a promising open research topic. Previous papers that relate mutation testing and performance have defined domain-specific mutation operators in Android [19] or model-based mutation operators [20,21,22]. There is also some incipient work in studying the applicability of traditional mutation operators to address non-functional properties [23]. We only found a paper that introduces the idea of using mutations to derive test suites for estimating the worst-case execution time [24]. To the best of our knowledge, the classical approach of mutation testing has not yet been applied with the intention of injecting mutations at the source-code level that affect the performance of the program.

In previous short papers [25, 26], we have explored the potential benefits and limitations of the application of mutation testing at the performance level, what we called performance mutation testing (PMT). Analogously to classic mutation testing, the goal of PMT is to generate variants of 
the original program (performance-mutants), where each variant models a performance bug, e.g. one that causes a noticeable degradation of the execution time of the program. These artificial faulty programs could then be used to assess and improve the effectiveness of performance tests. However, our initial findings suggest that PMT presents unique problems that make it far more challenging than mutation testing at the functional level. For example, contrary to functional mutation testing, mutants in PMT should exhibit a certain performance degradation while preserving the semantics of the original program. Interestingly, this means that semantically-equivalent mutants are actually the target of PMT, rather than an obstacle, as in traditional mutation. This could lead to problems with compiling optimization techniques, for example, which could automatically undo performance mutations if they detect that the original program and the mutant are equivalent [27].

In this article, we give a step forward in studying the feasibility of PMT. In particular, we first propose seven performance mutation operators based on classical performance bug patterns identified in the literature $[7,1,10,15,16,17,19,28,29]$. Then, we evaluate their effectiveness in assessing the fault-detection capability of test suites in three real-world $\mathrm{C}++$ programs. This required us to revisit the concept of valid, killed, alive and equivalent mutants. Among other results, we found that the new operators present greater potential to model useful performance faults than traditional operators. In particular, over $80 \%$ of performance-mutants generated in the programs under test (194 out of 241) were not detected by the functional test suite accompanying these applications. This is a positive aspect for performance-mutants because, by definition, mutants used in PMT are expected to preserve the internal state of the program. In contrast, the percentage of traditional mutants that are not detected by the functional test suite is much lower (42.7\%), which means that more than half of traditional mutants are directly discarded when applying PMT. We illustrate the usefulness of performance-mutants with specific examples extracted from the code of the programs under test. Finally, we give evidence that most of the proposed mutations are not invalidated by the optimizations implemented in compilers, a necessary condition for the feasibility of this approach.

The structure of the paper is as follows. Section 2 reviews the state of the art with regard to performance bugs and mutation testing. Section 3 motivates our work describing the challenges in PMT and Section 4 presents the research questions. The definition of performance mutation operators is addressed in Section 5. Section 6 describes the experimental evaluation and Section 7 shows and discusses respectively the results of the experiments to answer the research questions. Section 8 and 9 respectively present threats to validity and surveys related work focused on mutation testing and non-functional properties. Finally, Section 10 presents the conclusions and pending research questions to tackle in the future.

\section{BACKGROUND}

In the following subsections, we briefly describe the main terms used throughout the article.

\subsection{Performance bugs}

A performance bug arises when a program produces the correct result but its behavior suffers a noticeable degradation in terms of performance $[7,16]$. Therefore, a performance bug can be defined as a programming error that causes a significant performance degradation mainly due to the overuse 
of computational resources like execution time [30], memory [1] and energy [2, 3]. As an example, consider the real performance bug found in Groovy, illustrated in Figure 1. Lines 4-7 are only meaningful when the array argTypes is null or empty. However, those lines are always executed, wasting computation resources. To fix this, an if condition was added in line 3 to avoid lines 4-7 being executed unnecessarily [29].

According to the work by Jin et al. [7], there is a variety of common root causes that frequently lead to performance bugs, which include the inefficient combination of function calls, the unnecessary use of functions in certain contexts or synchronization issues. Well-tested applications such as Microsoft SQLServer, Apache HTTPD, and Mozilla Firefox, among others, are affected by hundreds of performance bugs [4,3]. Performance bugs can cause latency, a security breach in software systems and a disproportionate waste of computation resources. For these reasons, we have to pay special attention to detecting these kinds of issues and refactoring the code to achieve a version that meets the performance requirements of our system.

There exist widely-used techniques to detect and fix performance problems such as the supervised execution with profilers $[1,10,11]$ or the use of bug detection strategies that exploit known root causes for the appearance of performance bugs [7, 14, 15, 16]. However, performance bugs have usually been overlooked in traditional testing because they are more difficult to expose and detect than functional bugs since the former ones do not usually cause fail-stop symptoms [7]. The first meaningful studies on performance testing date back to the 90s [12]. Even though the interest in this field is growing in the last years [13, 28, 31, 32, 33], there is a lack of mechanisms to assess and improve the effectiveness of performance tests and it is acknowledged that most sophisticated performance testing strategies are required [8]. Considering all the above performance aspects, the detection of performance bugs presents numerous challenges to be addressed.

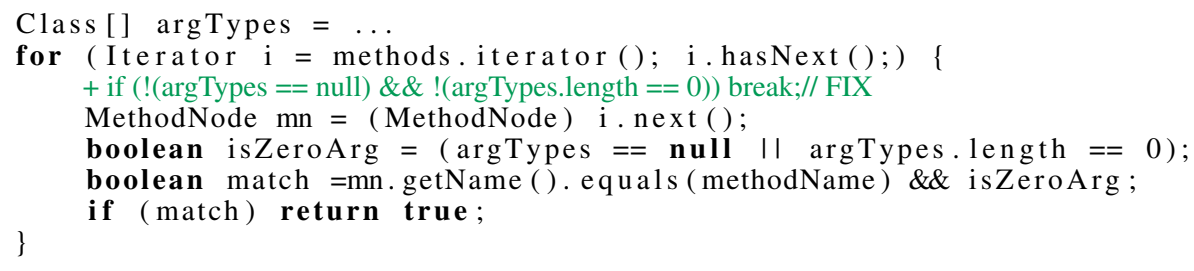

Figure 1. Real performance bug found in Groovy [29].

\subsection{Mutation testing}

Mutation testing is a structural testing method that aims to assess and improve the efficacy of test suites in discovering faults [18]. To do that, this technique injects faults (i.e., mutations) through a number of predefined rules (i.e., mutation operators) that help us to measure the adequacy of a test suite detecting them. Each of the new versions of the program, known as mutants, are then executed against the test suite to produce an output, in the same way as the original program. When a test case detects a mutant (i.e., the output of the mutant differs from the output of the original program), it is said that the mutant has been killed. Otherwise, the mutant remains alive. Ideally, a good mutation operator should produce mutants which are not easy to detect so that they can reveal weaknesses in a test suite. In contrast, it should minimize the generation of equivalent mutants (i.e., there is no 
input that can reveal the mutation). The problem of equivalent mutant identification together with the great number of mutants that can be produced are two stumbling blocks to a wider application of mutation testing.

This testing technique has been used in the past for testing software at different levels. Mutation testing has been applied to many programming languages at unit testing level (e.g., Java [34], C++ [35], C\# [36] or PHP [37], among many others), and has also been used at the integration level [38]. Additionally, we can find several works that propose the use of mutation testing at the specification level in applications such as web services [39], finite state machines [40] or timed automata $[33,41]$.

\section{PROBLEM STATEMENT}

The problem behind performance mutation testing (PMT) can be defined as finding a set of mutation operators that allow to assess and improve the fault-detection effectiveness of performance tests. Each mutation operator should perform a change in the source code of the program that causes a noticeable performance degradation while preserving the functionality of the original program. In this work, we transplant the application of traditional mutation testing to this new domain in order to assess the quality of performance tests: just like functional tests assess the compliance of the program with the functional requirements, performance tests indicate how close the program is to satisfy the non-functional specification. Since such specifications are not typically available, performance tests assess if the program under test deviates from the expected performance i.e., observing a significant performance degradation. Hence, the goal is to create faulty versions of the program which are close to the correct version of the program, in line with the two classic hypotheses behind mutation testing: the Competent Programmer Hypothesis and the Coupling Effect [42]. The former, also called Finite Neighborhood Hypothesis [43], assumes that programmers are competent, and therefore they tend to write programs close to the ideal version. In this new domain, it is important to clarify that this ideal version is not the optimal program in terms of performance (where program optimization techniques may be helpful) but one that does show the expected performance. The latter, i.e., the Coupling Effect, states that tests that are adequate to identify bugs introduced by simple changes should also be able to detect more complex faults. In this article, we hypothesize that these principles hold for PMT as they do for functional mutation testing on the basis of existing works in the literature describing performance bugs. For instance, Jin et al. [7] found that most of the studied performance bugs could be fixed through simple changes.

It could be argued that a simple way to introduce performance bugs is to insert harmless delays (e.g., Thread.sleep (100)) or to force unnecessary resource consumption (e.g., generation of unused objects) at convenient points in the program. However, although such a naive approach may be helpful in certain cases, it may not represent the types of faults introduced by programmers. Jin et al. [7] conducted an empirical study of 109 real-world performance bugs and concluded that almost $50 \%$ of performance bugs require both inputs with special characteristics and large-scale inputs to manifest. Similarly, Liu et al. [15] studied 70 real-world performance bugs in Android applications and found that bugs required a certain sequence of inputs to be perceived. Therefore, we assume 
that the performance bugs introduced with mutation should ideally mimic typical performance bugs made by programmers rather than simply synthetically consuming resources along the program.

In what follows, we define the classical terms related to mutation testing used throughout the article from a performance testing perspective, namely:

1. Performance-mutant. A variant of the original program where a syntactic change is introduced in the code to degrade the performance of the program. Those performancemutants preserving the semantics of the original program are referred to as valid performancemutants, while those changing the functionality will be labeled as invalid. The execution of the functional test suite on a performance-mutant is not a sufficient condition to determine whether that mutant is valid because the used test suite might not be complete enough to reveal a change in the functionality. Therefore, a performance-mutant that is not detected by the functional test suite is referred throughout the article to as potentially valid. Only a manual review can determine this condition with certainty.

2. Killed performance-mutant. A performance-mutant is killed if the tests can detect a noticeable performance degradation with respect to the performance observed in the original program. In this article, we use different thresholds to decide when a mutant is killed (cf. Section 6.2).

3. Alive performance-mutant. A performance-mutant is alive if it is not detected by our performance tests, but it is possible to design a feasible test input that uncovers the performance bug. By feasible we mean that the test input used to reveal the mutation is realistic for the program under test in a production environment. For instance, it could be the case that a performance mutation increased the execution time each time a line was read from a file: the more lines, the higher the degradation. However, that file may have a fixed number of lines not large enough to reveal the performance degradation in practice, and that situation should be assessed by the tester.

4. Equivalent performance-mutant. A performance-mutant is equivalent if there is no test input that can detect a noticeable increment between the observed performance in the original program and the mutant. Again, this definition depends on two factors: the thresholds and the feasibility of test inputs.

5. Performance mutation score. This metric allows assessing the fault-revealing capability of performance tests, measured as the ratio of killed performance-mutants to non-equivalent performance-mutants. By increasing this score, we improve the quality of the test suite and gain confidence that our software is free of performance bugs.

\section{RESEARCH QUESTIONS}

In this paper, we seek to evaluate the viability to apply PMT by defining domain-independent mutation operators (therefore, applicable to most programming languages) to assess and improve non-functional test suites. Namely, we aim at answering the following research questions in relation to PMT: 
Research question 1: How feasible is to define domain-independent performance mutation operators?

In this study, we aim at identifying some useful bug patterns to lead to plausible performance bugs (i.e., patterns that can potentially degrade the performance of the program), and studying their applicability in real-world programs.

Some recent papers have suggested the possibility of using traditional mutation operators in relation to performance [23, 25, 44]. By introducing traditional operators in our study, we want to shed light on whether these standard operators can model performance bugs or there is an actual need to incorporate new operators. To study the feasibility of using traditional operators for PMT, we address the following research question:

Research question 2: What percentage of mutants generated by traditional operators can model performance bugs?

Even if traditional operators produce effective mutants for our purpose, it is interesting to know whether the new mutation operators focused on performance are more effective than their traditional counterparts. Therefore, we aim to answer the following question:

Research question 3: How many performance-mutants do performance mutation operators generate and how often do these mutants change the functional behavior?

Once those performance-mutants have been executed against the test suite, we want to know whether those valid performance-mutants are able to cause a perceptible performance degradation and the kind of test inputs required to detect them. This is the aim of the next question:

Research question 4: Are the defined performance operators able to model some noticeable performance degradation?

Finally, in a previous work we noted the connection between PMT and the optimizations implemented in compilers. More specifically, we observed that both compiler optimizations and performance mutations seek to preserve the functionality of the program and, therefore, the transformations carried out by the former could potentially undo the latter. As a result, we complete the study with the following question:

Research question 5: Can optimizations performed by compilers revert the mutations injected with performance mutation operators?

\section{PERFORMANCE MUTATION OPERATORS}

In order to address RQ1, we first looked for real performance bug patterns that would inspire us to define performance-related mutation operators. Thus, we describe a set of mutation operators that 
Table I. Summary of performance mutation operators: source, abbreviation, category or main language features targeted, main performance property affected and need for preconditions.

\begin{tabular}{|c|c|c|c|c|}
\hline Source & Operator & Category & Performance issue & Preconditions \\
\hline$[7,29]$ & RCL & Loop perturbation & Execution time & $\checkmark$ \\
\hline$[16]$ & URV & Method call & Execution time & $\checkmark$ \\
\hline \multirow[t]{2}{*}[1,10]{} & MSL & Object generation; & Memory consumption & $\checkmark$ \\
\hline & & $\begin{array}{l}\text { Conditional statement; } \\
\text { Loop perturbation }\end{array}$ & Execution time & \\
\hline [7] & SOC & Conditional statement & Execution time & $\checkmark$ \\
\hline$[19,28]$ & HWO & Method call & Execution time & \\
\hline$[10,15]$ & CSO & Object generation & Memory consumption & \\
\hline$[10,17]$ & MSR & Collections & Memory consumption & \\
\hline
\end{tabular}

can be fixed by adding a condition inside the loop. Figure 1 shown in Section 2 depicts an example of this kind of performance bug.

Definition: Inspired by this bug pattern, the $R C L$ operator removes a stop condition in a loop so that the loop keeps iterating until another condition is satisfied. This is achieved by different means, namely 1) removing a break statement, 2) delaying a return statement with true or false, 3) removing a condition that uses a boolean variable expressly defined to stop the execution of the loop when the stop condition is met.

Example: The following code snippet shows the third option to apply the $R C L$ operator. The mutant removes the second condition $(! b)$ from the while loop, making the program to iterate while the first condition evaluates to true $(i<n)$. However, the loop could skip some iterations in the moment that $l[i]==1$.

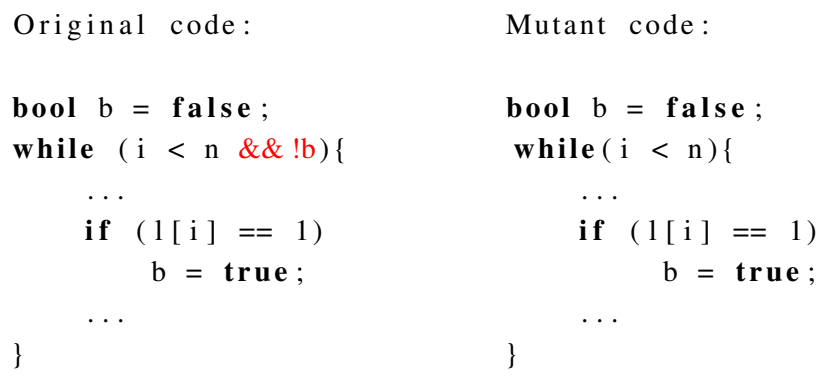

Preconditions: The $R C L$ performance-mutant can be generated when there are other conditions that can stop the loop at some point. This happens when the controlling expression of the loop is not empty (cases 1 and 2) or when there is more than one condition in the controlling expression of the loop (case 3).

\subsection{Unnecessary Recalculation of Values (URV)}

Motivation: Olivo et al. [16] found some situations where a program repeatedly iterates over a data structure that has not been modified between successive traversals. For instance, they identified the so-called ExtremeVal performance bug, which appears when the maximum or minimum element of a collection is calculated multiple times even though the collection never changes [16].

Definition: With the aim of generalizing the type of bug described in the motivation, the URV operator forces the recalculation of values previously computed. In more detail, this operator seeks for variables defined to store the value returned by an invocation to a method. Then, the variable is 
deleted and each reference to that variable is replaced by an invocation to the same method so that the computation performed by this method is repeated.

Example: The following fragment of code shows the deletion of the min variable and the replacement of the references to that variable by calls to the minValue function, forcing the recalculation of the minimum value of $v 12 \times \mathrm{n}$ times.

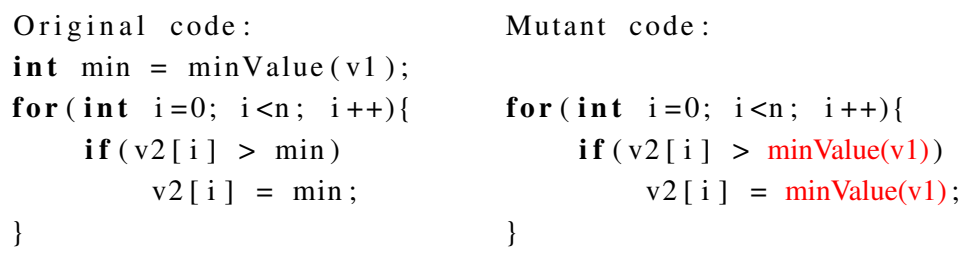

Preconditions: The $U R V$ performance-mutant can be generated when (1) there is a variable that stores a computed value and it is referenced more than once (case of equivalence), and (2) the variable that stores the computed value and the variables involved in its computation do not change between the point of the computation and the references to the variable.

\subsection{Move/Copy Statement into Loop (MSL)}

Motivation: Xu et al. [1] observed situations where many temporary objects were generated in a loop simply to provide a simple service (e.g., to assign a value to the fields of other objects). Yan et al. [10] also found that Never-Used and Rarely-Used Allocations (that is, objects that are created but hardly referenced) appear surprisingly often.

Definition: Based on the above performance bugs, the $M S L$ operator searches for parts of the code where new objects are generated before a loop statement. The operator then moves the object creation statement into the loop. In this way, the performance-mutant generates many temporary objects inside the loop instead of just one. Additionally, this operator moves conditional statements placed before loops' openings into the loops. This mutation can cause a significant degradation when the condition involves the execution of a costly operation that is repeated many times.

In some situations, $M S L$ has to copy the object or statement instead of moving it in order to preserve the syntax of the language. For instance, the if condition cannot be moved inside a loop when the conditional statement is an if/else statement; in that case, the if/else statement is preserved but the if condition is copied inside the loop.

Example: This example represents the mutation of moving an object creation statement inside a loop. In the performance-mutant, a new instance $o l$ is generated in each iteration, which results in the creation of many objects and the consequent waste of resources.

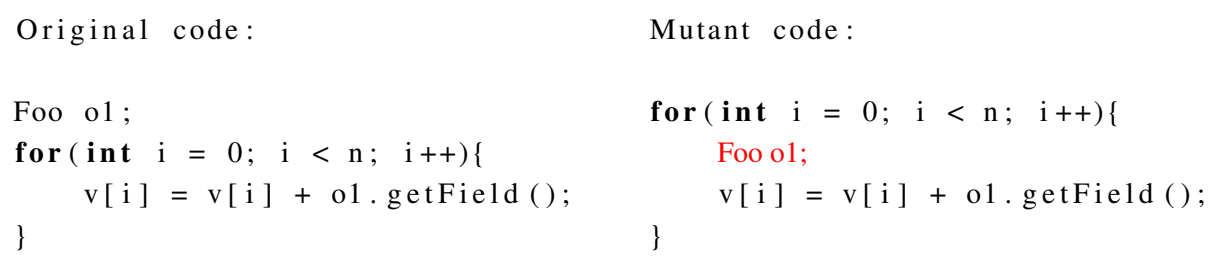

Preconditions: The $M S L$ performance-mutant can be generated when (1) the constructed object is not modified inside the loop, (2) none of the variables involved in the construction of the object changes inside the loop, (3) a conditional statement invokes at least one method or function, that is, 
the condition does not perform trivial operations (equivalence case), and (4) none of the variables involved in the conditions of the if statement changes inside the loop.

\subsection{Swap of Operands in Condition (SOC)}

Motivation: In cases where we have a multiple condition, the condition that takes more time to be evaluated is normally placed in the last position. This can avoid the evaluation of the most timeconsuming condition depending on the result of the first lighter conditions.

Definition: Considering the Skippable function bug pattern (see operator $R C L$ ) and reminding us of the traditional operator COR (Conditional Operator Replacement), the SOC operator forces the evaluation of a condition that might not be necessary depending on the result of other conditions. Thus, the $S O C$ operator swaps the operands in a condition linked by a binary logical operator (\&\& and $\|$ ) so that the most time-consuming condition is evaluated regardless of the other conditions.

Example: This example represents the compulsory evaluation and execution of the most costly method within the multiple conditions. As it can be seen in the original program, the invocation to costlyMethod can be saved when the condition $a !=1$ evaluates to true.

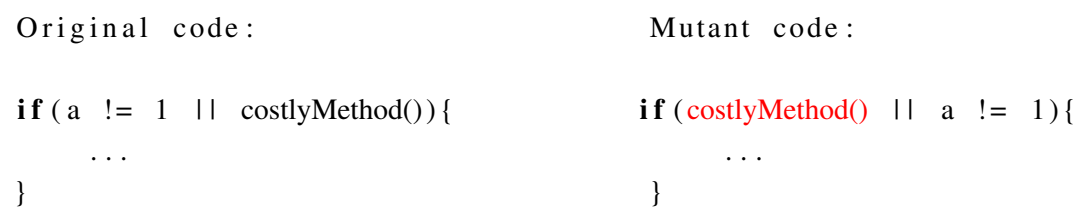

Preconditions: The SOC performance-mutant can be generated when (1) the condition invokes at least one method or function, that is, the condition does not perform trivial operations (case of equivalence), and (2) the most costly condition does not depend on the lighter conditions, such as in Figure 2.

\subsection{Simulation of Heavy-Weight Operation (HWO)}

Motivation: The study by Ongkosit and Takada [28] defines a list of potentially blocking operations in Android applications, such as bitmap processing and the access to network, storage, and databases. Linares-Vásquez et al. [19] defined three mutation operators to inject a long delay in some specific situations in Android applications too, like the insertion of a delay in the GUI listener thread. In general, some operations are more time-consuming than others and they can be especially problematic when they are contained in third-party libraries. That is, they can lead to developers' workload misunderstandings or they can vary the performance of a program depending on the context or the updates [10].

Definition: The $H W O$ operator injects a delay $t$ right after each invocation to methods in thirdparty libraries and known heavy-weight operations (storage access, network connection...) so that workload issues can be revealed. By default, the value of the delay $t$ could be correlated with the execution time of the original program.

Example: This example shows the insertion of a delay, in the form of sleep operation, just after a call to a costly method.

Original code: Mutant code:

costlyMethod(a1, a2); $\quad$ costlyMethod(a1, a2); 
sleep_for(std::chrono::seconds(t));

Preconditions: No preconditions are required by the $H W O$ operator.

\subsection{Creation of Short-lived Objects (CSO)}

Motivation: The idea behind the View Holder pattern is to reuse previously recycled items instead of generating new objects [15]. Similarly, the motivating example in Figure 1 in the study by Yan et al. [10] shows that in some situations we can reuse a single object across multiple calls to the same method.

Definition: Inspired by the works exposed in the motivation, the $C S O$ operator forces the generation of a new object every time a method is called instead of reusing an already created object. In detail, this operator targets the methods that receive an object as a parameter, which is used and returned by the same method. Then, the operator generates a clone of such objects, producing new short-lived objects inside the method every time the method is invoked.

Additionally, some languages provide other mechanisms to avoid the repetition of tasks that should be performed only once. Therefore, this operator could be adapted to generate more performance-mutants depending on the language of the application.

Example: This example represents the redundant creation of an object instance, $f 2$, when the existing object $f$ could be used.

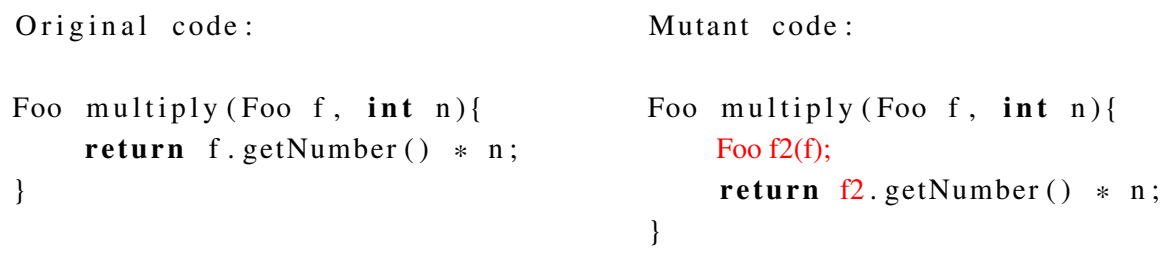

Preconditions: No preconditions are required by the $C S O$ operator.

\subsection{Memory Space Reservation (MSR)}

Motivation: Sometimes, the initial capacity of collections is not appropriate for the number of elements stored. Using small initial sizes can lead to frequent resize operations. In contrast, the pre-allocation of a collection at the maximal number of elements results in very low utilization.

Definition: Based on the Never-Used and Rarely-Used Allocations bug pattern [10] and on the causes of low utilization of collections [17], the MSR operator allocates less or more space for dynamic collections. This operator modifies a collection with dynamic allocation to shrink or expand the reservation space for elements to simulate both cases. The value of the size increase $t$ should be configured in the latter case.

Example: The example represents the two ways of applying the MSR operator: the space allocation for vector $v$ is reduced to 1 (first case), and it is increased by a factor of $t$, being $t>1$ (second case).

Original code:

vector $<$ Foo $>$ v (INIT_SIZE);

Original code:

Copyright () 0000 John Wiley \& Sons, Ltd.

Prepared using stvrauth.cls
Mutant code:

vector $<$ Foo $>$ v(1);

Mutant code : 
vector $<$ Foo $>$ v (INIT_SIZE); $\quad$ vector $<$ Foo $>$ v (INIT_SIZE $* \mathbf{t})$;

Preconditions: No preconditions are required by the MSR operator.

\section{EVALUATION}

In this section we describe the test subjects, the process followed to classify the mutants and the experimental procedure used to analyze the feasibility of applying PMT.

\subsection{Subjects under test}

We selected 3 open-source projects in $\mathrm{C}++$ that are distributed with functional test suites. Namely:

- TinyXML2 [45] (Txm): An efficient parser of XML files that can be integrated into other C++ programs to read and write XML documents. It contains 2,620 lines of code (LOC).

- XmlRPC++ [46] (Rpc): An XML-RPC protocol implementation to incorporate client-server communication through HTTP support into C++ applications. LOC: 2,194.

- Dolphin [47] (Dph): A navigational file manager used by KDE desktop applications. We have focused on the class KFileItemModel, which allows loading items of a directory. LOC: 2,771.

These programs were compiled and executed on a server running the operative system Ubuntu 14.04 and $32 \mathrm{~GB}$ of RAM memory.

For the evaluation of PMT with traditional mutation operators (RQ2), we selected the same subset of traditional operators that López et al. [23] proposed to optimize the code by reusing equivalent mutants:

- Arithmetic Operator Replacement (AOR).

- Relational Operator Replacement (ROR).

- Assignment Shortcut Replacement (ASR).

We used the mutation tool $M u C P P$ [35] for $\mathrm{C}++$ programs to apply these traditional operators. In the case of performance-mutants (RQ3 and RQ4), they were inserted manually into the programs due to the challenging task of automating these operators. To do so, in a first step, and having in mind each of the performance operators, we reviewed the code of the case studies searching for all the potential mutation locations. In a second phase, we analyzed in depth the list of locations and discarded those that did not meet the preconditions associated with these operators. Then, we applied the operators manually in the remaining locations to create the performance-mutants. Finally, we compiled the mutants to ensure that there were no invalid ones due to errors in the manual injection of mutations.

For the evaluation of the relation between PMT and compiler optimizations (RQ5), we applied the technique known as Trivial Compiler Equivalence or TCE [27]. This technique allows the automated detection of two equivalent programs when, as a result of the optimizations performed by compilers, the binary files of both programs turn out to be identical. In our work, we use the compiler $g c c$, a reliable compiler for all standards-compliant programs, to compare the binary files of the original 
program and the performance-mutants. The compiler invalidates a performance-mutant when there is no difference between its binary files and the ones of the original program.

\subsection{Experimental setup}

This evaluation seeks to analyze the feasibility of applying PMT using both traditional and performance mutation operators. As a proof of concept, we focused on the execution time as the measurable non-functional property to assess the generated mutants. The approach should extrapolate to other properties like memory by using proper profilers that help detect significant deviations in the memory usage when compared to the original program.

We reused the functional test suite developed for these programs and we adapted it to work as a performance test suite. To do that, we executed the original program 30 times and recorded the average execution time of each test case. These recorded times acted as test oracles in our experiments by adding new time-related assertions. In particular, we followed the steps described below:

1. First, we executed the functional test suite on each generated mutant. This allowed us to discard the mutants that were killed by the functional test suite and therefore were obviously not valid for PMT, since they did not preserve the semantics of the program. The remaining mutants, the ones used in our study, were labeled as potentially valid (see Section 3).

2. Then, we executed each test case on each potentially valid mutant, and recorded the execution time of each test case individually. This step was repeated 30 times to get the average time of each test case in each of these mutants.

3. Finally, we compared the mean time of each test case in the original program and each mutant. In this way, we could classify the mutants into killed and alive depending on whether they increased the execution time in any of the test cases.

In order to determine whether each mutant was killed or not we performed a hypothesis statistical test. Specifically, for each subject program and mutant, we stated a null and alternative hypothesis. The null hypothesis $\left(H_{0}\right)$ states that there is not a statistically significant difference between the execution times of the original program and the mutant, while the alternative hypothesis $\left(H_{1}\right)$ states that the execution time of the mutant is higher than the execution time of the original program and such difference is statistically significant. Specifically, a mutant is killed when the execution time of at least one test case is statistically higher compared with the execution time of that test case against the original program. Statistical tests provide a probability (named p-value) ranging in $[0,1]$. We used three different widely used p-value thresholds, namely $0.01,0.05$ and 0.1 under which results are considered to be statistically significant and are sufficient to reject the null hypothesis. Since the execution times collected do not follow a normal distribution (according to Shapiro-Wilk normality test), we used the Mann-Whitney U test for the analysis. Finally, we performed a manual review of the mutants that remained alive with $p$-value $<0.1$, in order to know how many of them were valid and whether we could find meaningful mutants.

Interruptions in the execution of a mutant caused by timeouts are normally easy to detect using either functional or non-functional test suites. Also, we cannot know whether the mutation will 
finally cause a change in the behavior. Therefore, we discarded those mutants causing a timeout in our evaluation.

\section{RESULTS}

In the following subsections, we report the results and how they answer the research questions:

\subsection{RQ1: Injection of performance mutations}

Table II shows the number of performance mutations that were injected in the subjects under test based on the performance mutation operators defined in Section 5. The results are divided by mutation operator and program. As it can be seen, all mutation operators could be applied in the conducted experiment, being Dolphin the program in which more mutations were injected. Additionally, all operators could be applied in two of these three programs (CSO and MSR did not lead to any mutants in TinyXML2). The mutation operators $U R V, S O C$ and $H W O$ were the most prolific operators, while we did not find many situations to apply CSO and MSL.

Table II. Performance-mutants divided by program and performance mutation operator

\begin{tabular}{lrrrrrrrr}
\hline Program & RCL & URV & MSL & SOC & HWO & CSO & MSR & Total \\
\hline Txm & 11 & 10 & 2 & 9 & 7 & - & - & 39 \\
Rpc & 10 & 25 & 1 & 23 & 13 & 1 & 2 & 75 \\
Dph & 9 & 41 & 4 & 34 & 12 & 11 & 16 & 127 \\
\hline Total & $\mathbf{3 0}$ & $\mathbf{7 6}$ & $\mathbf{7}$ & $\mathbf{6 6}$ & $\mathbf{3 2}$ & $\mathbf{1 2}$ & $\mathbf{1 8}$ & $\mathbf{2 4 1}$ \\
\hline
\end{tabular}

Regarding the application of the performance operators in these programs, we should remark the following particular cases:

- Operator $H W O$ : TinyXML2 reads and writes XML files using specific operations of a library to that end. Dolphin emits $Q t$ signals $^{\dagger}$ to intercommunicate objects. Finally, XmlRPC++ makes use of libraries to operate with sockets for client-server communication. The invocation to these functions has been the target of $H W O$. We set a generic delay of 1 second for these applications.

- Operator MSR: Dolphin employs $Q t$ dynamic containers and sometimes the program reserves memory for them through specific methods. Similarly, XmlRPC++ reserves memory for strings. This operator focuses on those operations. We set the value $t=2$ (i.e., we double the reserved size).

- Operator CSO: Dolphin and XmlRPC++ contain some methods that use static variables to reuse information between calls to the same method. These locations are the target of this operator.

Answer to RQ1: How feasible is to define domain-independent performance mutation operators? We have been able to define 7 mutation operators based on reported performance bugs in the

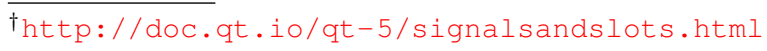


literature. Most of these operators require to preprocess the code to avoid the generation of some mutants that are known to alter the semantics of the program. Some other operators may require the configuration of some parameters prior to their application, as in the case of $H W O$ and MSR. Examples of performance-mutants based on all these mutation operators have been found in the three real-world programs, especially in the case of $U R V$ and SOC. The research questions RQ3 and RQ4 will explore whether these mutants can actually be useful to improve performance tests.

\subsection{RQ2: Analysis of traditional mutants}

Table III presents the results of the execution of traditional mutants to evaluate the test suite from a non-functional perspective. The table shows the number of generated mutants and how many of them are potentially valid mutants for PMT, and how many of them are killed and alive using the different thresholds of degradation. Recall that we removed mutants that raised the timeout, so the sum of killed and alive is not the same as the number of potentially valid mutants. The table also reports the mutants found as valid when the mutants that remained alive with $p$-value $<0.1$ were manually reviewed.

From 587 mutants generated in the three programs, 251 mutants passed the first step, that is, only $42.7 \%$ of the mutants survived the functional test suite execution. These results are graphically shown in Figure 3 divided by mutation operator. Taking into account that using traditional operators is costly, this low percentage is a detriment to the application of PMT using this set of operators.

Table III. Classification of mutants generated by the set of traditional operators (Mut.), potentially valid (Pot. valid), killed and alive mutants with $p$-value $<0.1, p$-value $<0.05$ and $p$-value $<0.01$, and valid mutants in the set of alive mutants with $p$-value $<0.1$ once these mutants were manually reviewed.

\begin{tabular}{|c|c|c|c|c|c|c|c|c|c|c|}
\hline \multirow{2}{*}{ Prog. } & \multirow[b]{2}{*}{ Mut. } & \multicolumn{2}{|c|}{ Pot. valid } & \multicolumn{2}{|c|}{$p$-value $<0.1$} & \multicolumn{2}{|c|}{$p$-value $<0.05$} & \multicolumn{2}{|c|}{$p$-value $<0.01$} & \multirow[b]{2}{*}{ Valid } \\
\hline & & $\#$ & $\%$ & Killed & Alive & Killed & Alive & Killed & Alive & \\
\hline Txm & 173 & 51 & $29.5 \%$ & 7 & 41 & 4 & 44 & 0 & 48 & 6 \\
\hline Rpc & 231 & 112 & $48.5 \%$ & 65 & 17 & 58 & 24 & 45 & 37 & 0 \\
\hline Dph & 183 & 88 & $48.1 \%$ & 78 & 5 & 77 & 6 & 60 & 23 & 0 \\
\hline Total & 587 & 251 & $42.7 \%$ & 150 & 63 & 139 & 74 & 105 & 108 & 6 \\
\hline
\end{tabular}

As expected, the number of killed mutants decreases as the threshold increases. In total, from the 105 mutants killed considering the most lenient threshold ( $p$-value $<0.1$ ), 45 of them appear as surviving mutants with the most stringent one $(p$-value $<0.01)$. We reviewed the live mutants (threshold 0.1) to analyze these mutants qualitatively. That set of live mutants accounts for $25.1 \%$ of the potentially valid mutants (63 out of 251). Even though it is difficult to determine whether these mutants are valid or not, it is likely that the functional test suite lacks specific test cases or asserts that are able to reveal most of these live mutants (57 out of 63). Indeed, it is also likely that many of the 150 killed mutants could also be detected using a better functional test suite. It is also remarkable that 30 mutants were detected with a timeout in $X m I R P C++$, which probably occurs because the mutation impedes the client-server communication.

We only found a few mutants from the set of alive mutants with $p$-value $<0.1$ that we believe that do not change the functional behavior. Figure 4 shows an example of one of those mutants generated by the mutation operator ROR in TinyXML2. As illustrated, the mutation causes the program to allocate memory even when the number of elements to be stored (cap) is equal to the memory currently allocated (_allocated). 


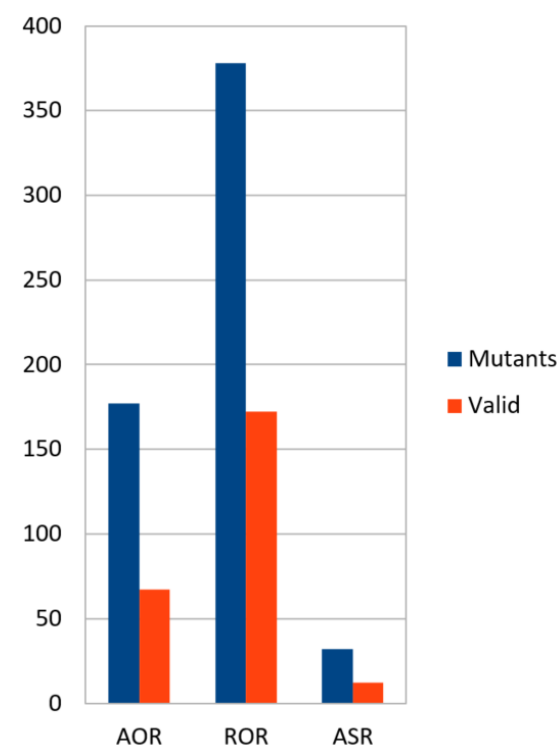

Figure 3. Number of mutants generated and potentially valid mutants by traditional mutation operator

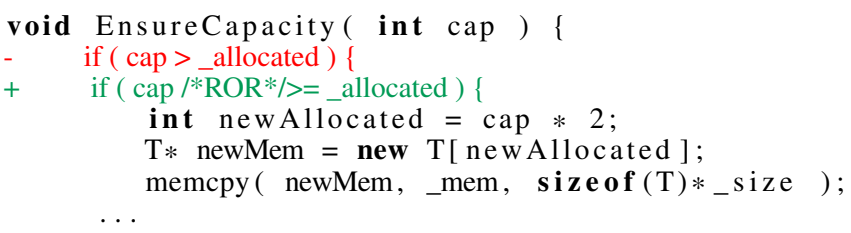

Figure 4. ROR mutant in TinyXML2

This situation is similar to the one identified by López et al. [23], where the change of relational operator produces that a skippable operation is executed.

Answer to RQ2: What percentage of mutants generated by traditional operators can model performance bugs? The three traditional operators selected (AOR, ROR and ASR) generate a large number of mutants, but only $42.7 \%$ of them are not detected by the functional test suite. Even though there is a considerable number of mutants that increased the execution time, a review of the live mutants reveals that few traditional mutations can really model meaningful performance bugs. As expected, these mutations appear in rare instances that are tied to the semantics of the program.

\subsection{RQ3: Analysis of performance-mutants}

Table IV shows the results of the execution of performance-mutants in a similar fashion to Table III.

At first sight, the most remarkable fact is that $80.5 \%$ of the generated performance-mutants passed the functional test suite (in contrast to $42.7 \%$ of the traditional mutants). The number of mutants generated with these 7 performance operators is not so high as with the 3 traditional operators (241 vs 587 mutants), which is a positive aspect in mutation testing.

The tendency regarding killed mutants is similar to that of traditional mutants. The percentage of live traditional mutants is higher than the percentage of live performance-mutants except for 
Table IV. Classification of performance-mutants generated by the set of performance mutation operators (Mut.), potentially valid (Pot. valid), killed and alive mutants with $p$-value $<0.1, p$-value $<0.05$ and $p$ value $<0.01$, and valid mutants in the set of alive mutants with $p$-value $<0.1$ once these mutants were manually reviewed.

\begin{tabular}{|c|c|c|c|c|c|c|c|c|c|c|}
\hline \multirow{2}{*}{ Prog. } & \multirow[b]{2}{*}{ Mut. } & \multicolumn{2}{|c|}{ Pot. valid } & \multicolumn{2}{|c|}{$p$-value $<0.1$} & \multicolumn{2}{|c|}{$p$-value $<0.05$} & \multicolumn{2}{|c|}{$p$-value $<0.01$} & \multirow[b]{2}{*}{ Valid } \\
\hline & & $\#$ & $\%$ & Killed & Alive & Killed & Alive & Killed & Alive & \\
\hline Txm & 39 & 24 & $61.5 \%$ & 11 & 9 & 8 & 12 & 4 & 16 & 8 \\
\hline Rpc & 75 & 56 & $74.6 \%$ & 24 & 24 & 17 & 31 & 3 & 45 & 20 \\
\hline Dph & 127 & 114 & $89.8 \%$ & 105 & 4 & 101 & 8 & 68 & 41 & 4 \\
\hline Total & 241 & 194 & $80.5 \%$ & 140 & 37 & 126 & 51 & 75 & 102 & 32 \\
\hline
\end{tabular}

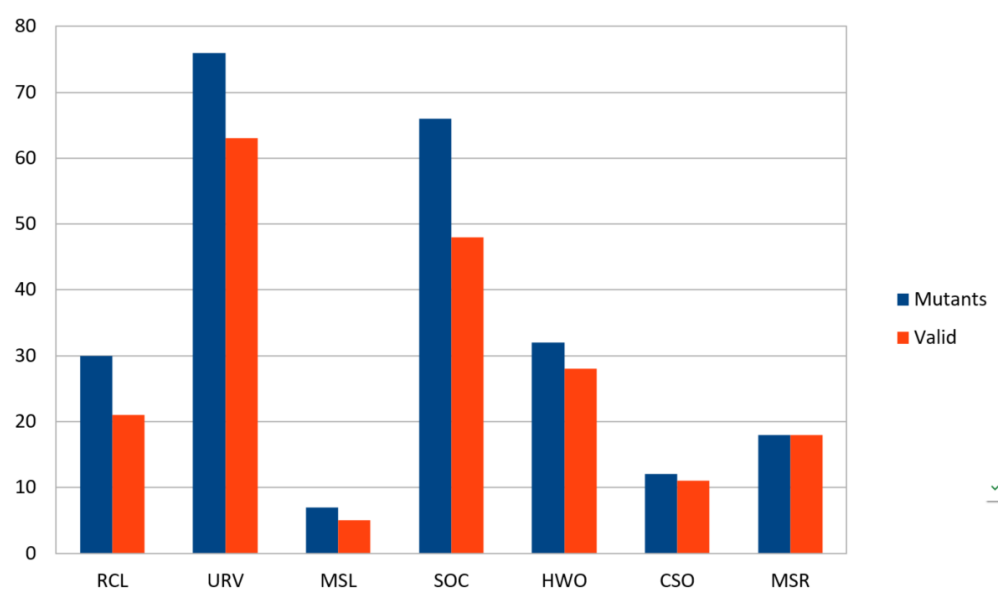

Figure 5. Number of mutants generated and potentially valid mutants by performance mutation operator

the threshold $p$-value $<0.01(50.7 \%$ vs $57,6 \%)$. A review process reveals that 32 out of those 37 performance-mutants that remained alive with $p$-value $<0,1$ do not change the functional behavior, and should be plausibly useful mutants to generate new test inputs. These numbers suggest that these performance-mutants are more difficult to detect than traditional ones, and may require special test scenarios beyond the ones typically designed to build functional test suites, which is in line with the observations in the literature (see Section 3). Only a few mutants change the functionality of the program. For example, a SOC mutation in XmlRPC++ swaps two invocations to the method nextTagIs, which modifies the value of the input-output parameter offset (see Figure 6). The program expects to find the tag PARAMS before PARAM in the response ( $<$ params $><$ param $>$ ). This mutation alters the semantics because the first call to nextTagIs updates the offset to the position of the tag PARAM, which makes impossible to find the tag PARAMS after that.

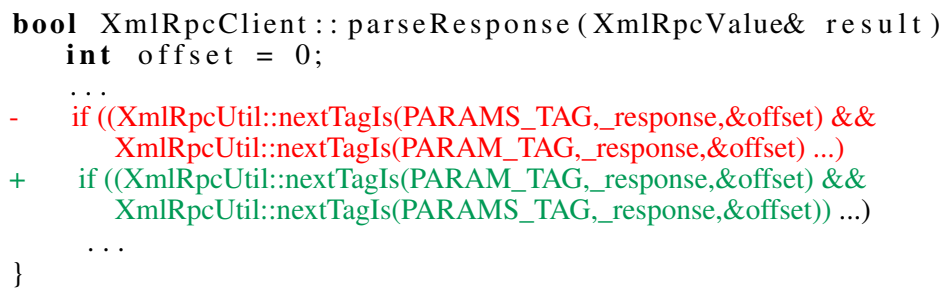

Figure 6. SOC mutant in $X m l R P C++$ 
Figure 5 presents a more detailed classification of the performance-mutants by mutation operator. The operator MSR was the only mutation operator where all the mutants generated are potentially valid for PMT, while $S O C$ is the one that more frequently alters the semantics of the program.

Answer to RQ3: How many performance-mutants do performance mutation operators generate and how often do these mutants change the functional behavior? The number of performance-mutants generated by the 7 performance operators defined is less than half of the mutants produced by the 3 traditional operators ( 241 vs 587 mutants). In principle, over $80 \%$ of those performancemutants do not change the functionality, which nearly doubles the percentage of potentially valid traditional mutants. A manual review of the performance-mutants that survived the execution of the non-functional test suite with $p$-value $<0.1$ reveals that a high percentage of those mutants $(86.5 \%)$ preserves the semantics of the program.

\subsection{RQ4: Qualitative analysis of significant performance mutations}

In the previous section, we manually reviewed the mutants that were alive with threshold $p$ value $<0.1$, and counted the number of them that could be classified as valid. In this section, we assess those mutants qualitatively in order to detect potentially helpful performance-mutants, even though we cannot say with certainty whether these mutants could be meaningful for testers of these programs.

There are several reasons why these mutants survived the test suite execution. Some of them simply require a test scenario that executes a fragment of code to reveal the performance issue. Some others can only be identified when executed on other platforms. For instance, XmIRPC++ applies some operations with sockets which are specific for Windows systems. However, in general, most of these mutants would require special and large-scale inputs to be detected.

Below, we describe some of the reviewed mutants that can cause noticeable performance failures.

$\boldsymbol{U} \boldsymbol{R} \boldsymbol{V}$ mutant: The method shown in Figure 7 returns the value of an attribute with a given name in an XML element. If such an attribute does not exist or its value is different from the one expected, it returns 0 . Since the method has to check for these conditions, the attribute is first searched by its name and saved in a reference variable $a$ (line 3 ). The search by name is performed by the method FindAttribute and it requires to iterate the list of attributes and compare their names with the one specified. In this mutant, each reference to the variable $a$ is replaced by a call to FindAttribute, which result in three searches instead of one (lines 6,10 and 12).

Thanks to the above mutant, we found that a test case that processed an XML file with a long list of attributes in its elements was missing to detect this performance issue. This is a clear example of the kind of inputs that would be required to reveal these issues.

MSR mutant: The fragment shown in Figure 8 presents a list (QList) of objects $K U r l$ (line 4). The size of the list is known in advance (itemCount) and the code reserves memory for that number of elements. The mutant disregards the prediction of the elements that will be appended (line 5), thereby causing a repetitive allocation of the data (resize operations) as the number of elements appended increases (line 8). 


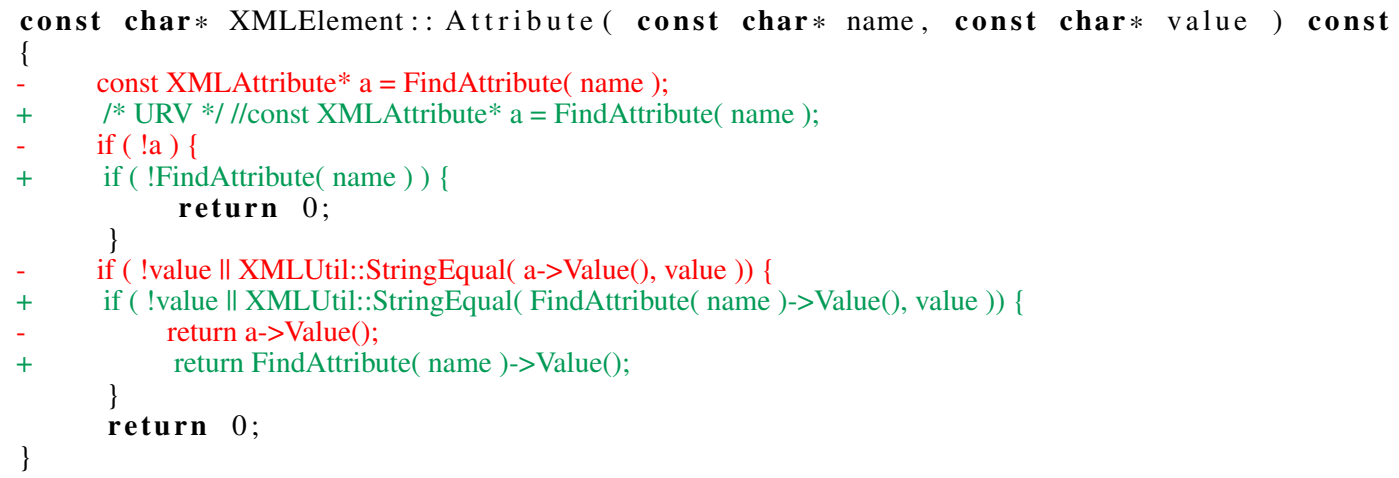

Figure 7. URV mutant in TinyXML2

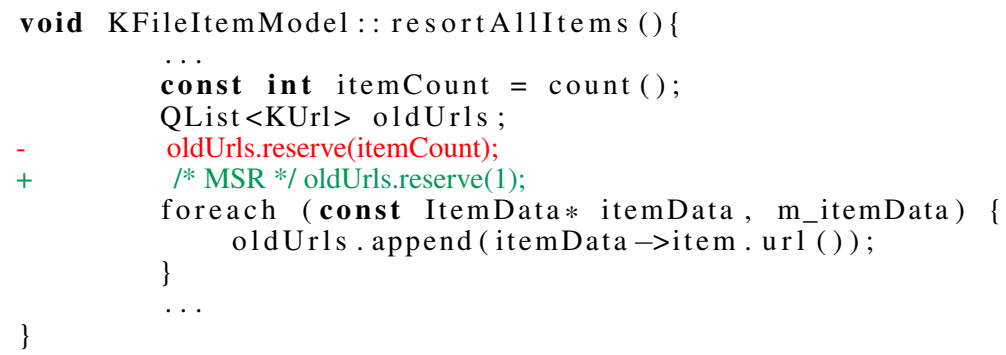

Figure 8. MSR mutant in Dolphin

This mutant should lead to the design of a test case that calls the method resortAllitems() when there is a large number of items in m_itemData.

RCL mutant: The code fragment presented in Figure 9 shows an interesting mutant generated by the operator $R C L$. The code shows a loop that looks for a specific entity to print a pattern. Once the entity has been found, the break statement avoids useless iterations. The mutant removes that statement, which leads to a performance issue when the method is called many times or if there are many entities.

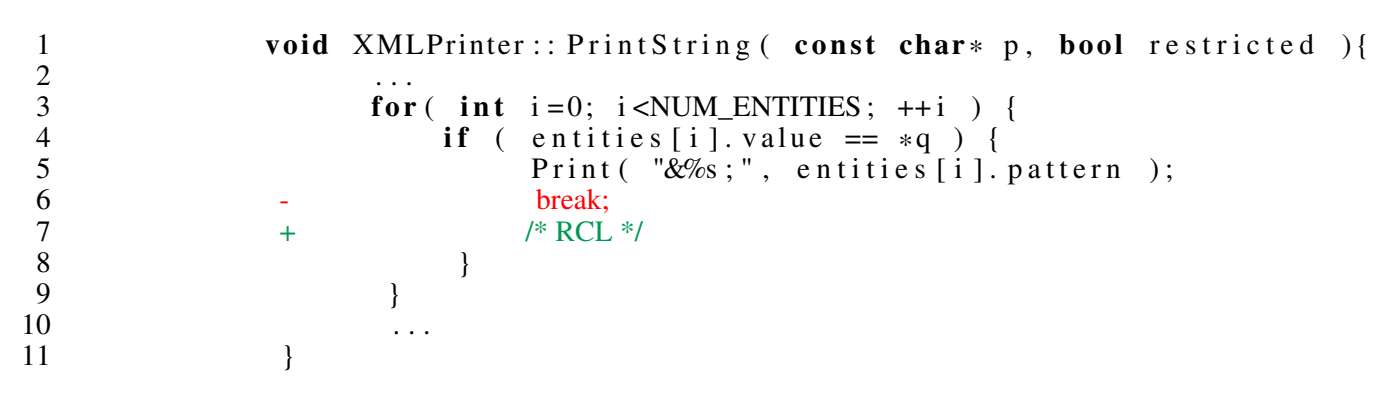

Figure 9. $R C L$ mutant in TinyXML2

SOC mutant: The method hasMember shown in Figure 10 searches for a member with a given name provided that the object is of type TypeStruct. The mutation swaps the check of the two 
conditions (name and type). This mutant forces the method to search for the member in a map container regardless of the type, thus increasing the execution time.

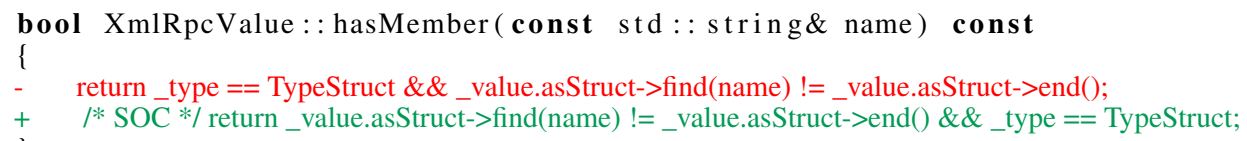

Figure 10. SOC mutant in XmlRPC++

This mutant should lead to the design of a test case that invokes this method several times from a XmlRpcValue object with_type different to TypeStruct and with the map container previously filled with several members.

Answer to RQ4: Are the defined performance operators able to model some noticeable performance faults? Yes. Even though we have found some performance-mutants that seem trivial to kill, we have also found some exemplary mutants extracted from the subjects under test that illustrate the kind of performance issues caused by the new mutation operators, and we have proposed new test scenarios to improve the current test suites.

\subsection{RQ5: Compiler optimizations}

Table V shows the results of the application of Trivial Compiler Equivalence (TCE) to performancemutants. We may recall that the objective was to check whether the optimizations performed by the compiler undo some of the changes introduced by the performance mutation operators. In particular, we compiled with no optimization settings and with the highest optimization level supported by the gcc compiler (-O3). As it can be seen, the results are similar in both cases: 8 mutants are reverted with the default option altogether in the three programs, and 10 mutants with level -O3. We have to note that the two performance-mutants reverted in TinyXML2 and all but the CSO mutant in $X m I R P C++$ corresponded to the same circumstance: the mutation was injected into a fragment of code that is activated in Windows systems through a preprocessor directive. Therefore, they were reverted by the compiler because we executed the experiments on a server running Ubuntu.

As a result, there are only two performance-mutants (generated by $U R V$ and $S O C$ ) invalidated by the compiler when applying the optimization level -O3. Figure 11 shows the reverted mutant generated by $S O C$, which swaps two similar conditions.

Table V. Number of mutants reverted by the compiler applying no optimization settings (None) and the highest optimization level (-O3). The results are divided by mutation operator and program under test.

\begin{tabular}{|c|c|c|c|c|c|c|}
\hline \multirow{2}{*}{ Operator } & \multicolumn{2}{|c|}{$T x m$} & \multicolumn{2}{|c|}{$R p c$} & \multicolumn{2}{|c|}{$D p h$} \\
\hline & None & -O3 & None & -O3 & None & $-\mathrm{O} 3$ \\
\hline RCL & - & - & - & - & - & - \\
\hline URV & - & - & - & 1 & - & - \\
\hline MSL & - & - & - & - & - & - \\
\hline SOC & - & - & - & - & - & 1 \\
\hline HWO & 2 & 2 & 5 & 5 & - & - \\
\hline MSR & - & - & - & - & - & - \\
\hline $\mathrm{CSO}$ & - & - & 1 & 1 & - & - \\
\hline Total & 2 & 2 & 6 & 7 & 0 & 1 \\
\hline
\end{tabular}




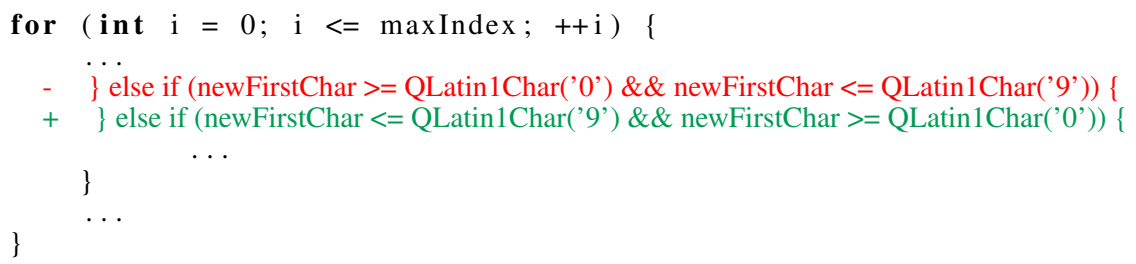

Figure 11. SOC mutant in Dolphin

Answer to RQ5: Can optimizations performed by compilers revert the mutations injected with performance operators? Only a couple of performance-mutants generated by the operators SOC and $U R V$ are invalidated applying TCE. These two mutants are only reverted by the compiler when an optimization level is set. There are also some other mutations injected by the operators $H W O$ and CSO that are not useful in our experiments because we use Ubuntu and these mutations can only be activated in Windows systems through preprocessor directives.

\subsection{Additional remarks}

The number of killed mutants was low in the experiments compared to traditional mutation, especially as the threshold increased. The fact that we adapted test suites focused on the functional aspect to work as performance test suites has probably prevented a higher number of performancemutants from being initially detected. An interesting aspect regarding equivalence in PMT is that the number of equivalent performance-mutants depends on the threshold. A coarse adjustment of the threshold could lead to many potentially valid mutants. However, with a fine-grained threshold, equivalent performance-mutants should not be frequent, which is a positive aspect in mutation testing. This factor also depends on the accuracy of the methods to measure the assessed performance properties. Additionally, and following the RIP model proposed by Ammann and Offutt [48], once the program has been infected (which in PMT implies a noticeable increase in the measured non-functional properties), the cases where that infection does not propagate to the output should be rare. In other words, it is unlikely that the effects of the infection are masked after the execution of the mutated statement, which is a possible cause for the appearance of equivalent mutants in traditional mutation testing.

Several papers in the past have evaluated the significance of mutation testing when applied in real scenarios $[49,50]$. These studies have been mostly performed using known traditional operators. Under the assumption that the hypotheses behind traditional mutation testing hold for PMT (see Section 3), we could expect that the benefits of applying the mutation-based approach hold for this new domain. However, we are still distant from being able to support this claim. First, we need to assess the level of automation that can be reached; then, only experiments with real case studies could give evidence of the ability of mutation-adequate performance tests to find performance bugs. 


\section{THREATS TO VALIDITY}

\section{Internal validity:}

The injection of performance mutations in the experimental evaluation has been done in a manual way, which is error-prone. We have proceeded with care to generate all possible performancemutants taking into account the constraints of each operator. Despite the manual injection of mutants, we have followed a systematic process consisting of four steps in the hope that it can be reproduced by any testers. Also, live performance-mutants have been reviewed manually to determine whether they could model interesting performance issues, being this process also prone to errors. We could have generated new test cases to kill performance-mutants. However, that would require both a deep knowledge of the program under test and the generation of large-scale inputs, which are not easy to design. There is the possibility that some of the mutants that survived the test suite could be detected with new functional test cases, but this would require the design of an adequate test suite, which would be a hard and time-consuming task.

\section{Construct validity:}

As mentioned earlier, the output of the executions in terms of time consumption is not deterministic and can be affected by the current computer workload or the specific characteristics of the machine. To mitigate this threat, we have compared 30 executions of each test case on the original program and each mutant.Additionally, we have performed a statistical analysis of the execution times and we have used three different thresholds ( $p$-values). More complex methods could be used, like the ones proposed by Reichelt and Kühne [9]. However, the methods that require many executions would make the application of the technique excessively expensive.

\section{External validity:}

The aim of our evaluation was to assess the feasibility of the definition and application of domainindependent performance operators, as this is the first evaluation of such operators at the sourcecode level as far as we know. As a proof of concept, we have used and analyzed three test subjects coded in $\mathrm{C}++$ language. Further experiments are required to know whether these results hold in other programs and programming languages. Also, we have used a subset of traditional operators in the evaluation, but there is the possibility that other traditional operators could be useful for PMT. Nevertheless, we have focused on three widely-used traditional operators that, additionally, have been proposed to optimize the code in terms of performance in a related work [23]. We have applied $g c c$ as the compiler to observe whether performance mutations could be reverted by compiler optimizations, as this is the more widely-used compiler for $\mathrm{C}++$ programs. However, new experiments should be conducted with compilers for other programming languages. 


\section{RELATED WORK}

\subsection{Specification-based mutation operators}

Mutation operators to test for timely behavior have been defined by Nilsson et al. [20] and AbouTrab et al. [51]. These operators mutate time constraints set in timed automata, where most of them focus on modifying the time boundaries of these constraints (i.e., increase or reduce each time property by a delta). Later, Lindstrom et al. [33] suggested to set the delta individually for each mutant generated with the operators defined by Nilsson et al. [20]; depending on the situation, a high delta would lead to the generation of trivial mutants (i.e., detected with simple test cases) and a low delta would lead to the creation of many equivalent mutants. Temple et al. [21] proposed the generation of variants of the program, called morphs, that modify the properties of software product lines and that can result in performance issues. Finally, Vega et al. [22] have recently performed a review focused on model-based mutation testing for timed systems.

\subsection{Domain-specific mutation operators}

A first strategy used to address the detection of performance bugs is the insertion of delays to test the responsiveness of applications [30]. However, the most common strategy to detect and fix bugs is to study real cases of bugs found in existing projects. This analysis can reveal patterns in the code that usually lead to this kind of bugs (i.e., bug patterns). Such bug patterns can then be automatically detected by means of rule-based detection using static analysis. Bug patterns related to the functional behavior have been collected by Pan et al. [14]. Similarly, bug patterns related to the performance behavior have been proposed by Jin et al. [7], Liu et al. [15], and Ongkosit and Takada [28] for Android applications. Recently, Linares-Vásquez et al. [19] defined a complete taxonomy of Android-related and Java-related performance bugs, which includes a subcategory of non-functional requirements.

All these studies have something in common: either they are focused on a specific language or context (such as Android [15] or desktop GUI applications [11]) or they describe specific bug patterns. For instance, Jin et al. [7] found that the function random should not be used by concurrent threads and that the function aggregateTransact should replace the invocations to doTransact when they are inside loops. While detecting and fixing these situations in the code can avoid specific performance issues, focusing only on these concrete cases is not effective in performance testing because we may miss some other kind of bugs that cause significant degradation. Additionally, the findings in that study are not applicable to other languages since those rules focus on specific functions only available in Java.

\subsection{Traditional mutation operators to address non-functional properties}

Program optimization to identify and fix some known performance issues is the target of a large body of research, from the diagnosis of energy inefficiency [2] to the improvement of the memory footprint [17]. Mutations have also been applied for optimization purposes. Langdon et al. [44] focused on software refactoring through genetic programming to optimize the performance of a program. Recently, López et al. [23] proposed using classical mutation operators applicable to different programming languages to find mutants with better non-functional aspects. However, the 
experiments in this paper show that it is unlikely that we can aspire to have a lot of useful mutants if we keep applying the same mutation operators that we have used so far to mimic behavioral faults. Indeed, the ratio of effective mutants may decrease as we add more traditional mutation operators, which is a problem taking into account the high cost of applying mutation testing. As a result of this issue, Sánchez et al. [26] suggested the use of an evolutionary algorithm to guide the search towards useful traditional mutants for the refinement of performance tests.

A recent research work presented by Lisper et al. [24] introduced the concept of targeted mutation, which aims at non-functional properties. This approach outlines the idea of introducing mutants to guide the generation and augmentation of test suites to be used for estimating the worst-case execution time. In contrast, our work pursues the definition and application of specific mutation operators affecting non-functional properties, such as the memory consumption or execution time among others.

There are some other works related to extra-functional properties that generate mutants with the sole aim of altering the system's functionality. For instance, Aichernig et al. [41] also focused on timed automata but changing different aspects of the syntax of the model instead of their time properties. Also, Wu et al. [52] proposed a set of mutation operators related to memory with the classical goal of perturbing the functional behavior of the program.

\section{CONCLUSION AND FUTURE WORK}

In this work, we have investigated the possibility to extend the current state of the art regarding mutation testing to evaluate and improve performance tests. The main finding of our study is that classical methods used in functional testing are not really effective when it comes to assess and improve non-functional tests, and that specific mutation operators and mechanisms to evaluate the outputs are necessary to successfully apply performance mutation testing. In particular, we have defined and evaluated seven new performance mutation operators that have highlighted the need for new performance tests. However, an analysis of a larger set of traditional operators would be desirable to confirm this finding, including those operators that usually produce a high number of equivalent mutants.

As far as we know, there are not many works that have tackled this research line, so much work lies ahead regarding the possible definition of new performance operators and the selection of the most effective ones. Also, its real effectiveness in practice and in different domains, or the best methods to detect performance issues associated with different non-functional properties should be further explored. Another challenge for future work is the automation of these performance operators. Static analysis techniques, used in other mutation tools like MuCPP [35], could be useful in a basic version of these operators. In a more advanced version, a data-flow analysis technique could be required to apply the preconditions required for some of these operators. LLVM's analysis passes [53] could be helpful to collect important information before applying the mutations. However, it is still unknown how costly such a method would be and whether it would compensate the number of mutants that would be actually avoided in real scenarios. Another point to consider is that it is unlikely that the effects of a mutation in PMT are masked, that is, most mutations should 
propagate to the output. As such, it is reasonable to think that the combination of mutations in highorder mutation could be more effective than first-order mutation. New experiments that compare both techniques could shed light about this issue in the future.

\section{ACKNOWLEDGEMENTS}

This work has been partially supported by the European Commission (FEDER), Spanish Government under MINECO projects DArDOS (TIN2015-65845-C3-3-R), FAME (RTI2018093608-B-C33), BELI (TIN2015-70560-R) and HORATIO (RTI2018-101204-B-C21) and the Operational Programme Andalusia under project APOLO (US-1264651).

\section{REFERENCES}

1. Xu G, Arnold M, Mitchell N, Rountev A, Sevitsky G. Go with the flow: Profiling copies to find runtime bloat. Proceedings of the 30th ACM SIGPLAN Conference on Programming Language Design and Implementation, PLDI '09, ACM: New York, NY, USA, 2009; 419-430, doi:10.1145/1542476.1542523. URL http: / / doi .org/10 . $1145 / 1542476.1542523$.

2. Liu Y, Xu C, Cheung SC, Lu J. Greendroid: Automated diagnosis of energy inefficiency for smartphone applications. IEEE Transactions on Software Engineering 2014; (1):1-1.

3. Nistor A, Jiang T, Tan L. Discovering, reporting, and fixing performance bugs. Working Conference on Mining Software Repositories, 2013; 237-246.

4. Zaman S, Adams B, Hassan AE. A qualitative study on performance bugs. IEEE Working Conference on Mining Software Repositories, 2012; 199-208.

5. URL https://econsultancy.com/site-speed-case-studies-tips-and-tools-for -improving-your-conversion-rate/, accessed: 14/10/19.

6. URL https://blog.kissmetrics.com/wp-content/uploads/2011/04/loading-time.pdf, accessed: $14 / 10 / 19$.

7. Jin G, Song L, Shi X, Scherpelz J, Lu S. Understanding and detecting real-world performance bugs. Proceedings of the 33rd ACM SIGPLAN Conference on Programming Language Design and Implementation, PLDI '12, ACM: New York, NY, USA, 2012; 77-88, doi:10.1145/2254064.2254075. URL http: / / doi .org/10.1145/ 2254064.2254075.

8. Molyneaux I. The Art of Application Performance Testing: Help for Programmers and Quality Assurance. O'Reilly Media, 2009.

9. Reichelt DG, Kühne S. How to detect performance changes in software history: Performance analysis of software system versions. Companion of the 2018 ACM/SPEC International Conference on Performance Engineering, ICPE '18, ACM: New York, NY, USA, 2018; 183-188, doi:10.1145/3185768.3186404. URL http: // doi .org/10 . $1145 / 3185768.3186404$.

10. Yan D, Xu G, Rountev A. Uncovering performance problems in Java applications with reference propagation profiling. Proceedings of the 34th International Conference on Software Engineering, ICSE '12, IEEE Press: Piscataway, NJ, USA, 2012; 134-144. URL http://dl . acm.org/citation. cfm?id=2337223. 2337240.

11. Jovic M, Hauswirth M. Listener latency profiling: Measuring the perceptible performance of interactive Java applications. Science of Computer Programming 2011; 76(11):1054 - 1072, doi:10.1016/j.scico.2010.04.009. URL https://doi.org/10.1016/j.scico.2010.04.009, special Issue on Principles and Practice of Programming in Java (PPPJ 2008).

12. Vokolos FI, Weyuker EJ. Performance testing of software systems. Proceedings of the 1st International Workshop on Software and Performance, ACM, 1998; 80-87.

13. Segura S, Troya J, Durán A, Ruiz-Cortés A. Performance metamorphic testing: Motivation and challenges. International Conference on Software Engineering: New Ideas and Emerging Results Track, 2017; 7-10. 
14. Pan K, Kim S, Whitehead EJ. Toward an understanding of bug fix patterns. Empirical Software Engineering Jun 2009; 14(3):286-315, doi:10.1007/s10664-008-9077-5. URL https : / / doi . org/10 . 1007 / s10664-008-9077-5.

15. Liu Y, Xu C, Cheung SC. Characterizing and detecting performance bugs for smartphone applications. Proceedings of the 36th International Conference on Software Engineering, ICSE 2014, ACM: New York, NY, USA, 2014; 1013-1024, doi:10.1145/2568225.2568229. URL http: / / doi . org/10.1145/2568225.2568229.

16. Olivo O, Dillig I, Lin C. Static detection of asymptotic performance bugs in collection traveresals. ACM SIGPLAN Conference on Programming Language Design and Implementation, 2015; 369-378.

17. Shacham O, Vechev M, Yahav E. Chameleon: adaptive selection of collections. ACM Sigplan Notices, vol. 44, ACM, 2009; 408-418.

18. Papadakis M, Kintis M, Zhang J, Le Traon Y, Harman M. Mutation testing advances: An analysis and survey. Advances in Computers 2017; .

19. Linares-Vásquez M, Bavota G, Tufano M, Moran K, Di Penta M, Vendome C, Bernal-Cárdenas C, Poshyvanyk D. Enabling mutation testing for Android apps. Proceedings of the 2017 11th Joint Meeting on Foundations of Software Engineering, ESEC/FSE 2017, ACM: New York, NY, USA, 2017; 233-244, doi:10.1145/3106237.3106275. URL http://doi.org/10.1145/3106237.3106275.

20. Nilsson R, Offutt J, Mellin J. Test case generation for mutation-based testing of timeliness. Electronic Notes in Theoretical Computer Science 2006; 164(4):97 - 114, doi:10.1016/j.entcs.2006.10.010. URL https://doi. org/10.1016/j.entcs.2006.10.010, proceedings of the Second Workshop on Model Based Testing (MBT 2006).

21. Temple P, Acher M, Jézéquel JM. Multimorphic testing. Proceedings of the 40th International Conference on Software Engineering: Companion Proceeedings, ICSE '18, ACM: New York, NY, USA, 2018; 432-433, doi: 10.1145/3183440.3195043. URL http: / / doi .org/10.1145/3183440.3195043.

22. Vega JJO, Perrouin G, Amrani M, Schobbens P. Model-based mutation operators for timed systems: A taxonomy and research agenda. 2018 IEEE International Conference on Software Quality, Reliability and Security (QRS), 2018; 325-332, doi:10.1109/QRS.2018.00045. URL https : / / doi .org/10.1109/QRS .2018.00045.

23. López J, Kushik N, Yevtushenko N. Source code optimization using equivalent mutants. Information and Software Technology 2018; 103:138 - 141, doi:10.1016/j.infsof.2018.06.013. URL https://doi.org/10.1016/j . infsof.2018.06.013.

24. Lisper B, Lindström B, Potena P, Saadatmand M, Bohlin M. Targeted mutation: Efficient mutation analysis for testing non-functional properties. 2017 IEEE International Conference on Software Testing, Verification and Validation Workshops (ICSTW), 2017; 65-68, doi:10.1109/ICSTW.2017.18.

25. Sánchez AB, Delgado-Pérez P, Segura S, Medina-Bulo I. Performance mutation testing: Hypothesis and open questions. Information and Software Technology 2018; 103:159 - 161, doi:https://doi.org/10.1016/j.infsof.2018. 06.015. URL 10.1016/j.infsof.2018.06.015.

26. Sánchez AB, Delgado-Pérez P, Medina-Bulo I, Segura S. Search-based mutation testing to improve performance tests. Proceedings of the Genetic and Evolutionary Computation Conference Companion, GECCO '18, ACM: New York, NY, USA, 2018; 316-317, doi:10.1145/3205651.3205670. URL http://doi.org/10.1145/ 3205651.3205670.

27. Papadakis M, Jia Y, Harman M, Le Traon Y. Trivial compiler equivalence: A large scale empirical study of a simple, fast and effective equivalent mutant detection technique. Proceedings of the 37th International Conference on Software Engineering - Volume 1, ICSE'15, IEEE Press: Piscataway, NJ, USA, 2015; 936-946, doi:10.1109/ICSE.2015.103. URL http://dx.doi.org/10.1109/ICSE.2015.103.

28. Ongkosit T, Takada S. Responsiveness analysis tool for Android application. Proceedings of the 2Nd International Workshop on Software Development Lifecycle for Mobile, DeMobile 2014, ACM: New York, NY, USA, 2014; 1-4, doi:10.1145/2661694.2661695. URL http: // doi.org/10.1145/2661694.2661695.

29. Nistor A, Chang P, Radoi C, Lu S. Caramel: Detecting and fixing performance problems that have non-intrusive fixes. 2015 IEEE/ACM 37th IEEE International Conference on Software Engineering, vol. 1, 2015; 902-912, doi: 10.1109/ICSE.2015.100.

30. Yang S, Yan D, Rountev A. Testing for poor responsiveness in android applications. 2013 1st International Workshop on the Engineering of Mobile-Enabled Systems (MOBS), 2013; 1-6, doi:10.1109/MOBS.2013.6614215.

31. Han S, Dang Y, Ge S, Zhang D, Xie T. Performance debugging in the large via mining millions of stack traces. 2012 34th International Conference on Software Engineering (ICSE), 2012; 145-155, doi:10.1109/ICSE.2012.6227198.

32. Zhang P, Elbaum S, Dwyer MB. Automatic generation of load tests. Proceedings of the 2011 26th IEEE/ACM International Conference on Automated Software Engineering, ASE '11, IEEE Computer Society: Washington, DC, USA, 2011; 43-52, doi:10.1109/ASE.2011.6100093. URL http://dx.doi.org/10.1109/ASE.2011. 6100093. 
33. Lindström B, Offutt J, Gonzalez-Hernandez L, Andler SF. Identifying useful mutants to test time properties. 2018 IEEE International Conference on Software Testing, Verification and Validation Workshops (ICSTW), 2018; 69-76, doi:10.1109/ICSTW.2018.00030.

34. Ma YS, Offutt J, Kwon YR. MuJava: an automated class mutation system. Software Testing, Verification and Reliability 2005; 15(2):97-133.

35. Delgado-Pérez P, Medina-Bulo I, Palomo-Lozano F, García-Domínguez A, Domínguez-Jiménez JJ. Assessment of class mutation operators for $\mathrm{C}++$ with the MuCPP mutation system. Information and Software Technology 2017; 81:169 - 184, doi:10.1016/j.infsof.2016.07.002. URL https://dx.doi.org/10.1016/j.infsof. 2016.07.002.

36. Derezińska A. Quality assessment of mutation operators dedicated for C\# programs. 2006 Sixth International Conference on Quality Software (QSIC'06), 2006; 227-234, doi:10.1109/QSIC.2006.51. URL https://dx. doi.org/10.1109/QSIC.2006.51.

37. Shahriar H, Zulkernine M. Mutec: Mutation-based testing of cross site scripting. Proceedings of the 2009 ICSE Workshop on Software Engineering for Secure Systems, IEEE Computer Society, 2009; 47-53.

38. Delamaro ME, Maldonado JC, Mathur AP. Interface mutation: An approach for integration testing. IEEE Transactions on Software Engineering May 2001; 27(3):228-247.

39. Lee SC, Offutt AJ. Generating test cases for XML-based web component interactions using mutation analysis. Proceedings of the 12th International Symposium on Software Reliability Engineering (ISSRE'01), Hong Kong, China, 2001; 200-209.

40. Fabbri SPF, Delamaro ME, Maldonado JC, Masiero P. Mutation analysis testing for finite state machines. Proceedings of the 5th International Symposium on Software Reliability Engineering, Monterey, California, 1994; 220-229.

41. Aichernig BK, Lorber F, Ničković D. Time for mutants — model-based mutation testing with timed automata. Tests and Proofs, Veanes M, Viganò L (eds.), Springer Berlin Heidelberg: Berlin, Heidelberg, 2013; 20-38.

42. Offutt AJ. Investigations of the software testing coupling effect. ACM Transactions on Software Engineering Methodology Jan 1992; 1(1):5-20, doi:10.1145/125489.125473. URL http://doi.org/10. $1145 / 125489.125473$.

43. Gopinath R. On the Limits of Mutation Analysis. Dissertation, Oregon State University jun 2017. URL http: //hdl.handle.net/1957/61528.

44. Langdon WB, Harman M. Optimizing existing software with genetic programming. IEEE Transactions on Evolutionary Computation 2015; 19(1):118-135.

45. TinyXML2. https://github. com/leethomason/tinyxm12. Accessed: 14/10/19.

46. XmlRPC++. http://xmlrpcpp. sourceforge.net/. Accessed: 14/10/19.

47. Dolphin. https://www.kde.org/applications/system/dolphin. Accessed: 14/10/19.

48. Ammann P, Offutt J. Introduction to Software Testing. 1st edn., Cambridge University Press: New York, NY, USA, 2008.

49. Daran M, Thévenod-Fosse P. Software error analysis: A real case study involving real faults and mutations. SIGSOFT Software Engineering Notes May 1996; 21(3):158-171, doi:10.1145/226295.226313. URL http: //dx.doi.org/10.1145/226295.226313.

50. Delgado-Pérez P, Habli I, Gregory S, Alexander R, Clark J, Medina-Bulo I. Evaluation of mutation testing in a nuclear industry case study. IEEE Transactions on Reliability Dec 2018; 67(4):1406-1419, doi:10.1109/TR.2018. 2864678.

51. AbouTrab MS, Brockway M, Counsell S, Hierons RM. Testing real-time embedded systems using timed automata based approaches. Journal of Systems and Software 2013; 86(5):1209 - 1223, doi:10.1016/j.jss.2012.12.030. URL https://doi.org/10.1016/j.jss.2012.12.030.

52. Wu F, Nanavati J, Harman M, Jia Y, Krinke J. Memory mutation testing. Information and Software Technology 2017; 81:97-111, doi:10.1016/j.infsof.2016.03.002. URL https://doi.org/10.1016/j. infsof.2016.03.002.

53. LLVM's passes. https://llvm.org/docs/Passes.html. Accessed: 14/10/19. 